\title{
Temporal requirements for ISL1 in sympathetic neuron proliferation, differentiation, and diversification
}

Qingquan Zhang (1)', Ru Huang', Youqiong Ye², Xiaoxia Guo', Jun Lu³, Fugui Zhu², Xiaohui Gong', Qitong Zhang ', Jie Yan', Lina Luo', Shaowei Zhuang ${ }^{4}$, Yihan Chen ${ }^{1}$, Xiaodong Zhao ${ }^{3}$, Sylvia M. Evans ${ }^{5}$, Cizhong Jiang $\mathbb{1}^{2}$, Xingqun Liang ${ }^{1}$ and Yunfu Sun (iD)

\begin{abstract}
Malformations of the sympathetic nervous system have been associated with cardiovascular instability, gastrointestinal dysfunction, and neuroblastoma. A better understanding of the factors regulating sympathetic nervous system development is critical to the development of potential therapies. Here, we have uncovered a temporal requirement for the LIM homeodomain transcription factor ISL1 during sympathetic nervous system development by the analysis of two mutant mouse lines: an $/ s / 1$ hypomorphic line and mice with $/ s / 1$ ablated in neural crest lineages. During early development, ISL1 is required for sympathetic neuronal fate determination, differentiation, and repression of glial differentiation, although it is dispensable for initial noradrenergic differentiation. ISL1 also plays an essential role in sympathetic neuron proliferation by controlling cell cycle gene expression. During later development, ISL1 is required for axon growth and sympathetic neuron diversification by maintaining noradrenergic differentiation, but repressing cholinergic differentiation. RNA-seq analyses of sympathetic ganglia from Is/1 mutant and control embryos, together with ISL1 ChIP-seq analysis on sympathetic ganglia, demonstrated that ISL1 regulates directly or indirectly several distinct signaling pathways that orchestrate sympathetic neurogenesis. A number of genes implicated in neuroblastoma pathogenesis are direct downstream targets of ISL1. Our study revealed a temporal requirement for ISL1 in multiple aspects of sympathetic neuron development, and suggested Is/1 as a candidate gene for neuroblastoma.
\end{abstract}

\section{Introduction}

Neurons of the sympathetic nervous system (SNS) innervate visceral organs, glands, and blood vessels, and play important roles in maintaining homeostasis. Aberrant development of the SNS causes neurocristopathies associated with cardiovascular instability, gastrointestinal dysfunction, as well as neuroblastoma that arises from developing sympathetic neurons ${ }^{1}$. The SNS is derived

\footnotetext{
Correspondence: Cizhong Jiang (czjiang@tongji.edu.cn) or Xingqun Liang (xingqunliang@tongji.edu.cn) or Yunfu Sun (yfsun@tongji.edu.cn)

${ }^{1}$ Key Laboratory of Arrhythmia, Ministry of Education, East Hospital, Tongji University School of Medicine, Shanghai, China

${ }^{2}$ School of Life Sciences and Technology, Tongji University, Shanghai, China

Full list of author information is available at the end of the article Edited by $\mathrm{G}$. Raschellà
}

from trunk neural crest (NC) cells. As NC cells commit to the sympathetic lineage, they downregulate the expression of genes characteristic of NC cells such as Sox10, and express several sympathetic neuron-specific genes such as Phox $2 b$ and markers of catecholamine biosynthesis (TH, $\mathrm{DBH})$. The newly generated sympathetic neurons transiently exit the cell cycle, and then reenter the cell cycle and are proliferating. Thereafter, an increasing number of sympathetic neurons exit the cell cycle, which peaks at embryonic day 14.5 (E14.5), and undergo post-mitotic differentiation ${ }^{2-5}$.

Bone morphogenetic protein (BMP) and Notch signals are required for induction and coordination of early sympathetic differentiation and progenitor maintenance. 


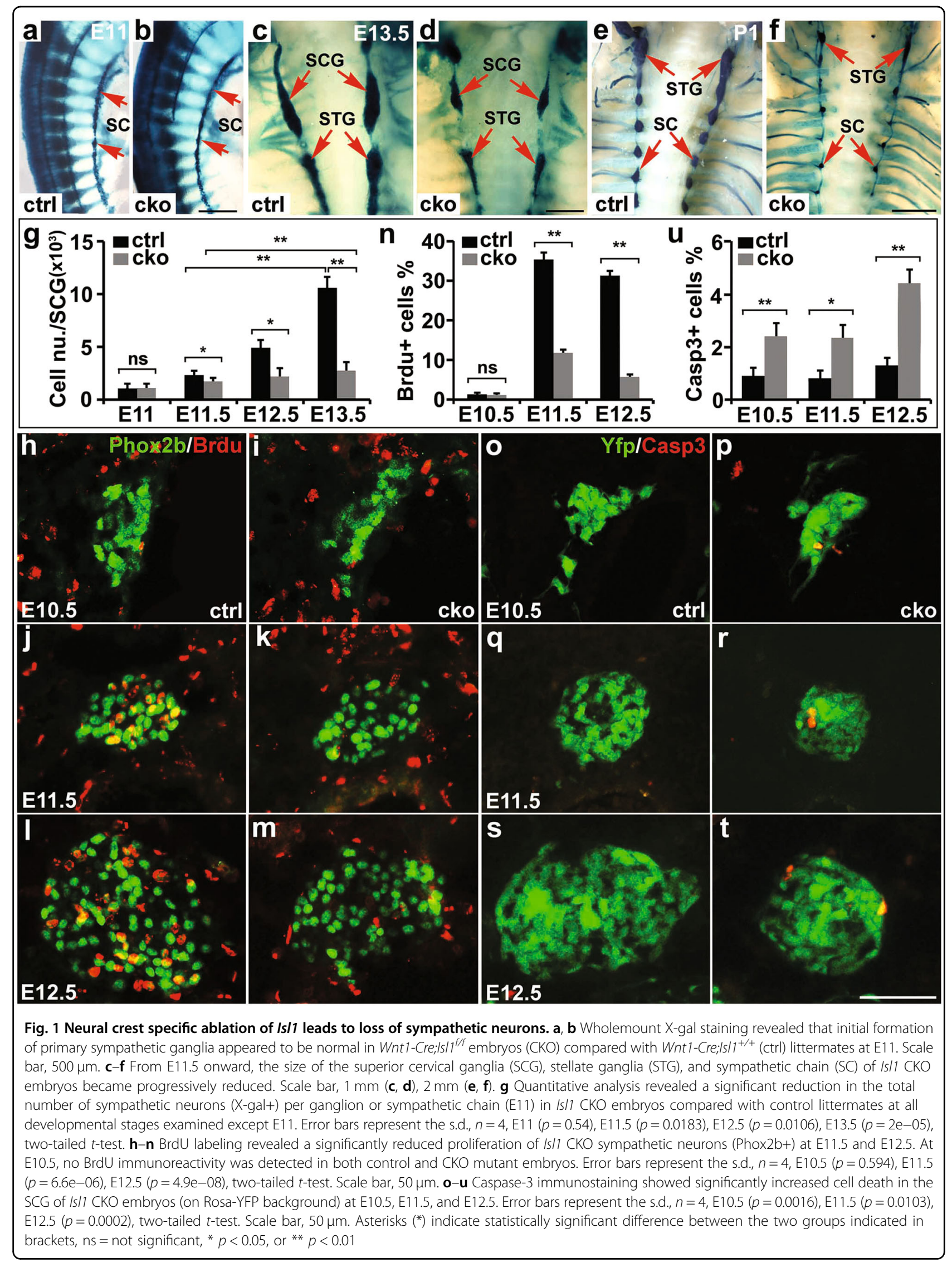


Enhanced Notch signaling results in increased Sox10+ progenitors at the expense of differentiated neurons in sympathetic ganglia ${ }^{6-9}$. BMPs initiate sympathetic neurogenesis by inducing sequential expression of a number of transcription factors ${ }^{2-4}$, including Phox2a/2b, Mash1, Hand2, Gata2/3, and Insm1. In Phox2b knockout mice, NC progenitors fail to initiate sympathetic differentiation and express Hand2, Gata3, TH, and $\mathrm{DBH}^{10}$. Gata2/3, Insm1, and Hand2 are BMP-induced transcription factors essential for sympathetic neuron proliferation and differentiation downstream of Mash1 and Phox2b. Together, these factors constitute an early transcriptional network essential for sympathetic neuron development ${ }^{2-4}$.

Segregation of initially bimodal sympathetic neurons into distinct noradrenergic and cholinergic neurons occurs relatively later during development, and is controlled by intrinsic as well as target derived signals ${ }^{11,12}$. Recent studies have uncovered a cross-repressive and maintenance mechanism for sympathetic neuron diversification, which involves antagonistic actions between pro-cholinergic factors Ntrk3 (TrkC)/Ret/Tlx3 and pro-noradrenergic factors Hmx1/Ntrk1 (TrkA) ${ }^{13,14}$. TrkC and Ret repress Hmx1 expression and induce cholinergic differentiation. Conversely, $\mathrm{Hmx} 1$ represses Tlx3 and Ret, induces TrkC, and maintains noradrenergic phenotypes.

ISL1 is an LIM-homeodomain transcription factor expressed in all peripheral neurons ${ }^{15}$. ISL1 plays an essential role in sensory neuron differentiation by activating genes critical for sensory neuron phenotype, and repressing genes expressed earlier during sensory neuron development ${ }^{15}$. ISL1 is required for early sympathetic neuron development ${ }^{16}$; however, genes and signaling pathways regulated by ISL1 and direct targets of ISL1 in sympathetic neurons remain unexplored. In this study, we uncovered a temporal requirement for ISL1 in various aspects of SNS development. ISL1 regulates directly or indirectly a number of genes essential for sympathetic neuron proliferation and differentiation, many of which have been implicated in neuroblastoma pathogenesis, suggesting $I s l 1$ as a candidate gene for neuroblastoma.

\section{Results}

ISL1 is required for proliferation and survival of sympathetic neurons at early developmental stages

We performed wholemount X-gal staining of Wnt1-Cre; Isl1 ${ }^{\mathrm{f} / \mathrm{f}} ;$ Rosa26-LacZ mutant (CKO) and Wnt1-Cre;Isl1 ${ }^{+/+}$; Rosa26-LacZ control (ctrl) embryos, and found that primary sympathetic column/chain of Isl1 CKO and ctrl embryos appeared comparable up until E11 (Fig. 1a, b). Thereafter, however, the size of Isl1 CKO sympathetic ganglia became progressively reduced (Fig. 1c-f). At E10.5-11, the number of sympathetic neurons was comparable between Isl1 CKO and control embryos (Fig. 1g).
However, from E11.5 to E14.5, the number of Isl1 CKO sympathetic neurons was significantly decreased compared to controls (Fig. 1g).

Sympathetic neurons pause cell division at E10.5, and then reenter the cell cycle from E11.5 onward, until their terminal differentiation during later development ${ }^{5}$. Consistent with this, at E10.5, we observed few, if any, proliferating sympathetic neurons (BrdU+/Phox $2 \mathrm{~b}+$ ) in either Isl1 CKO or control sympathetic ganglia (Fig. 1h, i, and n). At E11.5-12.5, however, we observed a significant reduction in the number of BrdU+ cells in Isl1 mutant sympathetic ganglia compared to controls at E11.5 ( 11\% vs. $35 \%$ ) and E12.5 ( 7\% vs. 30\%) (Fig. 1j-n). In addition, cleaved Caspase-3 immunostaining revealed a significant increase in cell death in Isl1 CKO sympathetic ganglia at E10.5-E12.5 (Fig. 1o-u).

\section{RNA-seq analysis reveals downregulation of a large number of genes essential for cell cycle progression at early developmental stages}

To better understand the genetic program regulated by ISL1, we performed RNA-seq analysis on sympathetic ganglia of Isl1 CKO and control embryos at E11.5-12, a stage when minimal cell loss occurred in Isl1 CKO sympathetic ganglia. We identified 358 downregulated and 555 upregulated transcripts in Isl1 CKO mutants (Ifold change Isl1 CKO vs. ctrl $\mid \geq 1.5, p \leq 0.05$ ) (Fig. 2a and Supplementary Table 1). Gene Set Enrichment Analysis (GSEA) revealed significant downregulation of gene sets representing the cell cycle, response to DNA damage stimulus, and sympathetic neuron development, but upregulation of gene sets representing $\mathrm{NC}$ development and glial differentiation (Fig. 2b-f). Selected cell cycle genes were verified by qPCR on FACS sorted sympathetic neurons from E11.5 Isl1 CKO and control embryos (Rosa26-tdTomato+). These included, e.g., Mki67 (all phases), Ccnd1 (G1-S phase), Smc2/4, Bub1 (M phase), Arhgap11a, Pbk (proliferative signaling) (Fig. 2g). Reduced expression of Ccnd1 and Mki67 was further confirmed by immunostaining (Fig. 2h-m).

\section{ISL1 is required for effective downregulation of NC and glial progenitor factors during initial sympathetic neuronal differentiation \\ Our RNA-seq and qPCR analyses revealed significant} upregulation of genes associated with NC (Sox10, Foxd3, Snai1, Sox2, and Crabp2) and glial (Fabp7, Metrn, Mpz, and Phgdh) progenitors (Fig. 3a and Supplementary Table 1), suggesting a failure in their developmental downregulation in sympathetic neuroblasts, and an increase in gliogenesis. To investigate the role of ISL1 in early sympathetic neurogenesis, we further analyzed coexpression of Sox10 (NC and glial progenitors, and sympathetic neuronal progenitors), Phox $2 b$ (sympathetic 


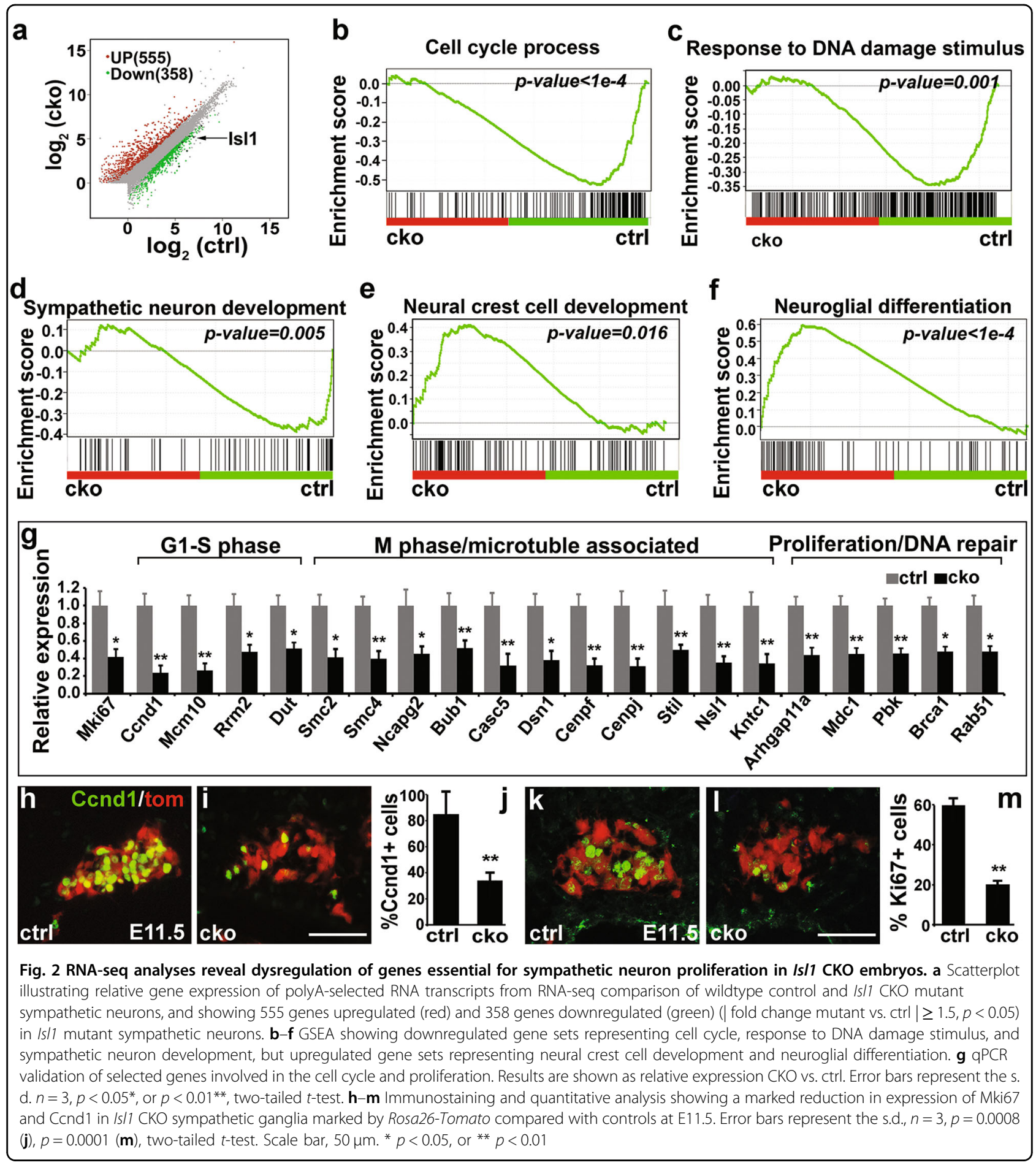

progenitors and neurons), and ISL1 during early sympathetic development. At E10.5 and 11.5, a proportion of sympathetic progenitors co-expressed Phox $2 \mathrm{~b}$ and Sox10; however, few, if any, ISL1-expressing or Isl1-Cre lineagelabeled sympathetic neurons co-expressed Sox10 (0-1 per section) (Fig. 3b-d, arrow). At E11.5, all ISL1expressing sympathetic neurons co-expressed Phox $2 \mathrm{~b}$; however, a subset of Phox $2 b+$ cells did not express ISL1 and were presumably progenitors for $\mathrm{NC}$, sympathetic neurons, or glia (Fig. 3e, arrow). In Isl1 CKO sympathetic ganglia, the number of Phox $2 b+$ sympathetic neurons was decreased; however, the number of Sox $10+$ cells, and Sox $10+/$ Phox $2 b+$ progenitors was significantly increased (Fig. 3f-h). 


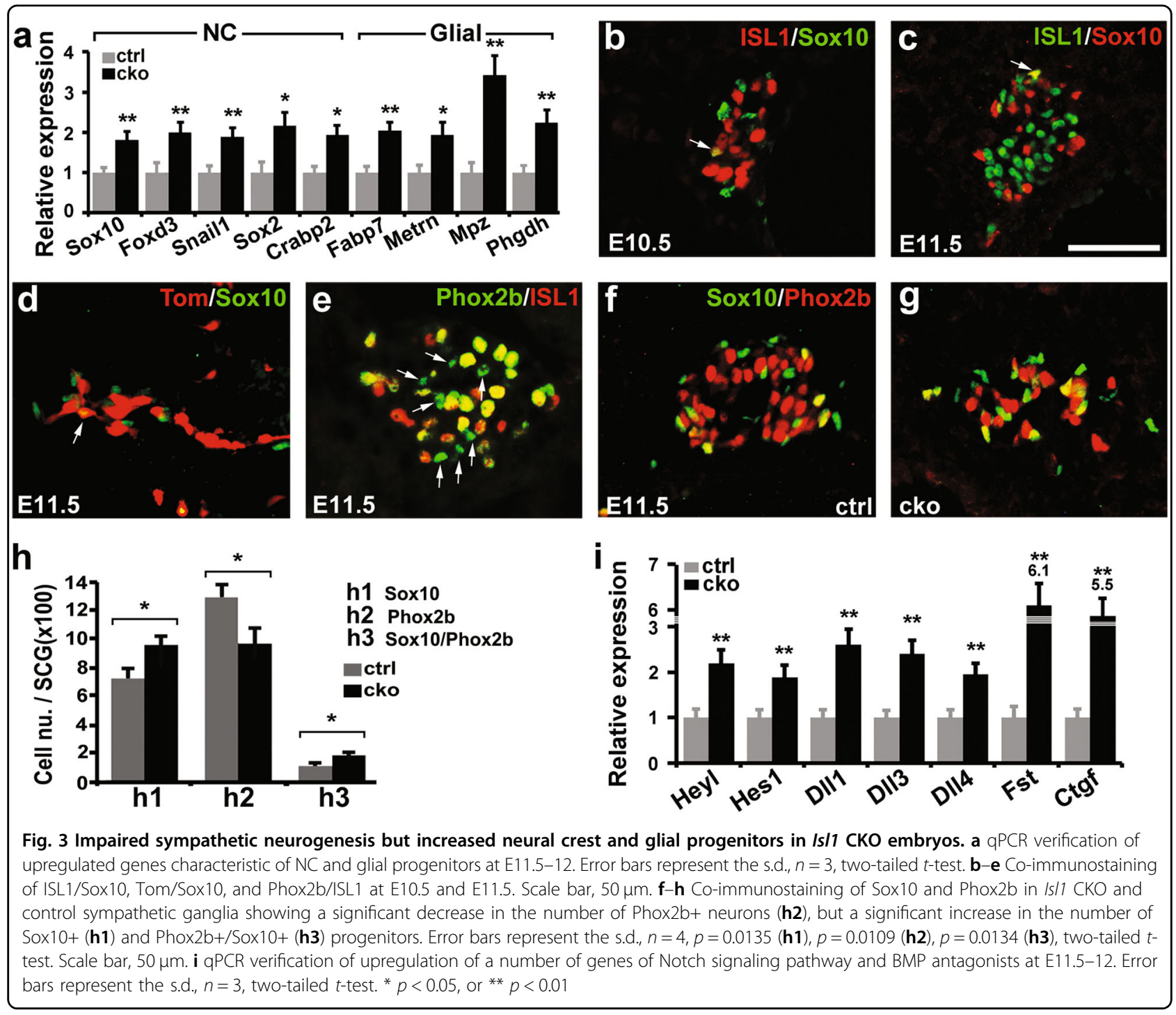

BMP and Notch signals are required for coordinating sympathetic differentiation and progenitor maintenance $^{6-9}$. We found that ablation of Isl1 during early sympathetic development resulted in reduced BMP signaling (Bmpr1b), but upregulated BMP antagonist signaling (Ctgf and Fst), and a significant increase in expression of Notch ligands (Dll1, Dll3) and downstream target genes (Hes1, Heyl, and Sox2) ${ }^{17}$ (Fig. 3i and Supplementary Table 1). Together, these data suggest that ISL1 plays an important role in initial sympathetic neurogenesis, likely by modulating BMP and Notch signaling pathways.

Role of $I s / 1$ in the gene regulatory network essential for sympathetic neuron differentiation at early developmental stages

Our RNA-seq data revealed significant downregulation of genes involved in neuronal development. We further examined significantly downregulated genes for those that might contribute to the observed phenotypes of Isl1 CKO SNS, confirming downregulation in their expression by qPCR on E11.5 sympathetic neurons (Fig. 4a). These included genes involved in transcriptional regulation (e.g., Gata2/3, Insm2, Tlx3), chromatin modification (e.g., Dnmt1, Kdm6b), and RNA binding (Elavl2/3, Lin28a/b), many of which are known to play critical roles in sympathetic neuron development ${ }^{2-4}$ (Fig. 4a). Downregulation of selected genes was further confirmed by in situ hybridization or by immunostaining (Fig. 4b-m).

To understand the role of ISL1 in sympathetic axon development and neurotransmission, we examined sympathetic innervation by $\mathrm{TH}$ immunostaining. We observed a marked reduction or nearly absence of sympathetic innervations in the heart at E14.5 and E17.5 (Supplementary Figure 1a-d) and the salivary gland at P1 (Fig. 4o) in Isl1 CKO mice compared to controls. 


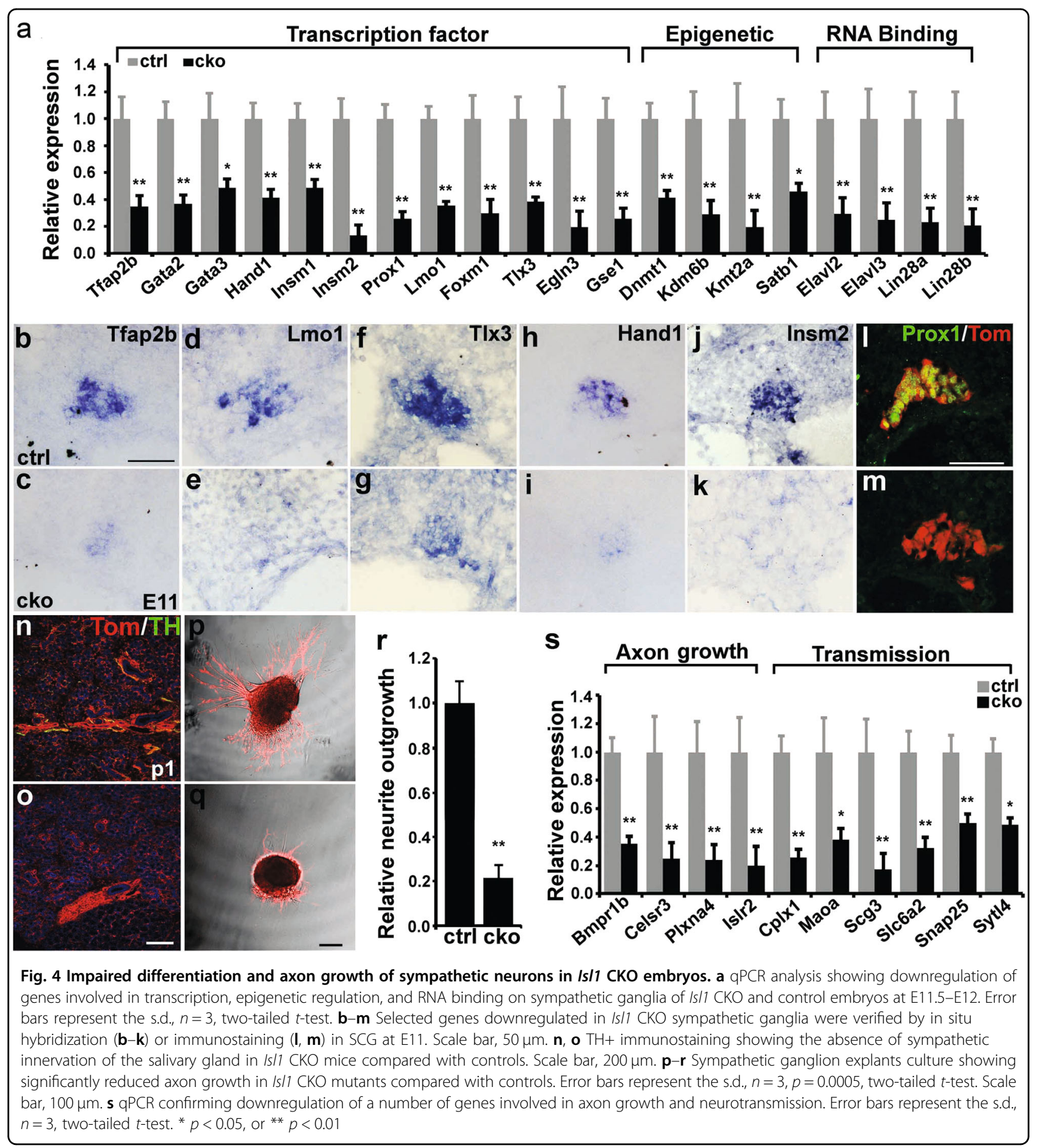

However, defects in sympathetic innervation may be secondary to neuronal loss in Isl1 CKO sympathetic ganglia. Therefore, we cultured sympathetic ganglia from E12 Isl1 CKO and control mice, and axon growth was assessed after $24-48 \mathrm{~h}$. We observed a significant 5-fold reduction in neurite outgrowth in Isl1 CKO mutants compared to controls (Fig. 4q, r). Furthermore, RNA-seq analysis revealed significantly altered expression of genes involved in neuronal projection morphogenesis and function. qPCR confirmed downregulation of genes involved in axon growth (e.g., Bmpr1b, Plxna4), neurotransmission (e.g., Cplx1, Scg3, Snap25), and altered expression of genes of neurotransmitters or related enzymes (Fig. 4s; Supplementary Figure 1e and f). 


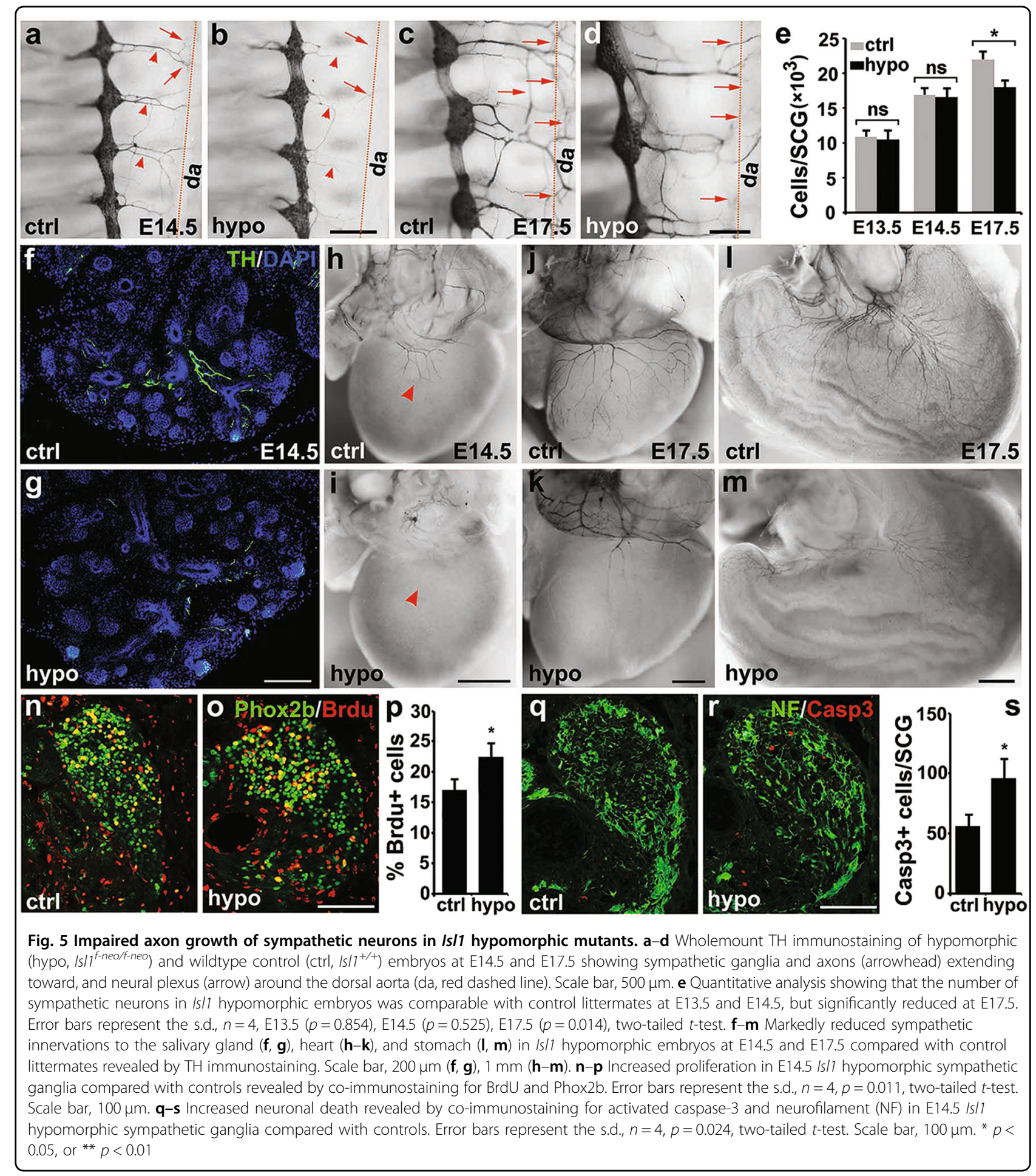

Reduced $I s / 1$ expression leads to impaired sympathetic innervation, and delayed cell cycle withdrawal during later development

Marked cell loss in Isl1 CKO sympathetic ganglia occurred well before robust sympathetic neuronal differentiation, precluding the study of the role of ISL1 during later sympathetic neuronal development. Therefore, we analyzed a mouse line with reduced Isl1 expression (hypomorphic $^{18}$ (Supplementary Figure 2a-e). TH immunostaining revealed that the size of sympathetic ganglia and the number of neurons in Isl1 hypomorphic embryos were not significantly altered prior to E17.5, 
although a reduction in sympathetic neuron number was observed in Isl1 hypomorphic mice at E17.5 (Fig. 5a-e). This allowed us to examine the role of ISL1 during later developmental stages.

In control embryos, neurites of sympathetic ganglia began to extend branches to innervate the dorsa aorta (da, dashed line) at E14.5, and formed a complex nerve plexus around the dorsal aorta at E17.5 (Fig. 5a, c, arrow). In Isl1 hypomorphic embryos, neurites of sympathetic ganglia appeared to be shorter with fewer branches at E14.5, and failed to form the aortic plexus at E17.5 (Fig. 5b, d, arrow). Similarly, sympathetic innervation to other target organs such as the salivary gland, heart, and stomach were markedly reduced in Isl1 hypomorphic embryos at E14.5 and E17.5 (Fig. 5f-m).

At E14.5-16.5, we observed significantly increased neuronal proliferation (BrdU+/Phox2b+) in Isl1 hypomorphic sympathetic ganglia (Fig. $5 \mathrm{n}-\mathrm{p}$ ). However, at E12.5-13.5, proliferation of sympathetic neurons was comparable between Isl1 hypomorphic and control mice (Supplementary Figure 3), suggesting delayed cell cycle withdrawal of Isl1 hypomorphic sympathetic neurons during later development. In addition, we observed significantly increased cell death, which may account for the overall loss of sympathetic neurons in Isl1 hypomorphic mutants (Fig. 5q-s).

\section{RNA-seq analysis revealed dysregulation of genes important for axon growth and neurotransmission in $/ s / 1$ hypomorphic sympathetic neurons during later development}

We performed RNA-seq analysis on Isl1 hypomorphic and wildtype sympathetic ganglia at E14.5 and identified 641 upregulated and 526 downregulated genes $(p<0.05)$ (Fig. 6a and Supplementary Table 2). GO analysis revealed that downregulated genes were over-represented in categories such as neuron development and neurotransmission, whereas upregulated genes were mainly cell cycle related (Fig. 6b, c; Supplementary Table 2). Similarly, GSEA analysis revealed downregulation of gene sets representing neuron differentiation and axonogenesis, but upregulation of a gene set representing the cell cycle in Isl1 hypomorphic embryos (Fig. 6d-f). Selected genes involved in axon growth, neurotransmission, and the cell cycle were verified by qPCR (Fig. 6g, h).

Intersection of the two RNA-seq data sets from Isl1 $\mathrm{CKO}$ and hypomorphic mutants identified only 84 genes commonly regulated by ISL1 at early ( $9.2 \%$ overlap) and later $(7.1 \%)$ developmental stages (Supplementary Figure 4). The commonly downregulated genes (46) were over-represented in categories such as protein secretion and synaptic vesicle transport, and upregulated genes (38) were involved in regulation of apoptosis (Supplementary Figure 4). Consistent with observed phenotypes, a subset of cell cycle-related genes were activated by ISL1 at early developmental stages but failed to downregulate in Isl1 hypomorphic sympathetic neurons at later developmental stages. Another subset of genes involved in protein secretion and nervous system development were repressed early but activated later by ISL1 during sympathetic neuron development (Supplementary Figure 4). These results suggested a distinct temporal requirement for ISL1 during sympathetic neuron development.

\section{ISL1 is required for diversification of noradrenergic and cholinergic sympathetic neurons}

RNA-seq and GSEA analyses revealed downregulation of a gene set representing noradrenergic differentiation, but upregulation of a gene set representing cholinergic differentiation in Isl1 hypomorphic sympathetic neurons (Fig. 7a, b; Supplementary Table 3). These included cholinergic genes (Ret, Tlx3, Sst, and Vip) and noradrenergic genes (Hmx1, Lmo1, Ntrk1, and TH) ${ }^{19}$. Alterations in their expression were confirmed by qPCR, in situ and immunostaining (Fig. 7c-r). At E14.5 and E17.5, Tlx3, Vip, and Sst were upregulated, but Hmx1, Lmo1, and Ntrk1 were downregulated in Isl1 hypomorphic sympathetic ganglia. Ret expression was transiently upregulated at E14.5, but back to normal level at E17.5, whereas expression of Ntrk3, Chat, and Vacht was relatively normal at E14.5 but became upregulated at E17.5. Conversely, TH expression was unchanged at E14.5, but downregulated at E17.5. These data suggested that reduced ISL1 led to a failure both in repression of a cholinergic gene program and in maintenance of a noradrenergic program.

\section{ISL1 directly regulates a number of genes required for sympathetic development}

To gain an insight into the direct downstream targets of ISL1 in sympathetic neurons, we performed ISL1 ChIPseq analyses on E12.5-14.5 sympathetic ganglia. Analyses of these data revealed 3031 ChIP-seq peaks for ISL1, which also contained other motifs for Phox2a, Nkx6.1, Lhx3, Nanog, and Gata3 (Fig. 8a, b; Supplementary Table 4 and 5). Spatial annotation of ISL1 binding sites revealed 2046 potential ISL1 targets, including those involved in neuron differentiation and axonogenesis (Fig. 8c, Supplementary Figure 5a and Table 4). Intersection of ChIP-seq data with RNA-seq data from Isl1 CKO and hypomorphic mutants revealed 130 (71 downregulated, 59 upregulated) and 186 (86 downregulated, 100 upregulated) genes that were directly regulated by ISL1 during early and later sympathetic development, respectively (Fig. 8d, g; Supplementary Table 6 and 7). For direct targets of ISL1 downregulated in E12 Isl1 CKO mutants, over-represented categories included neurotransmission and cell-cell signaling, whereas upregulated 


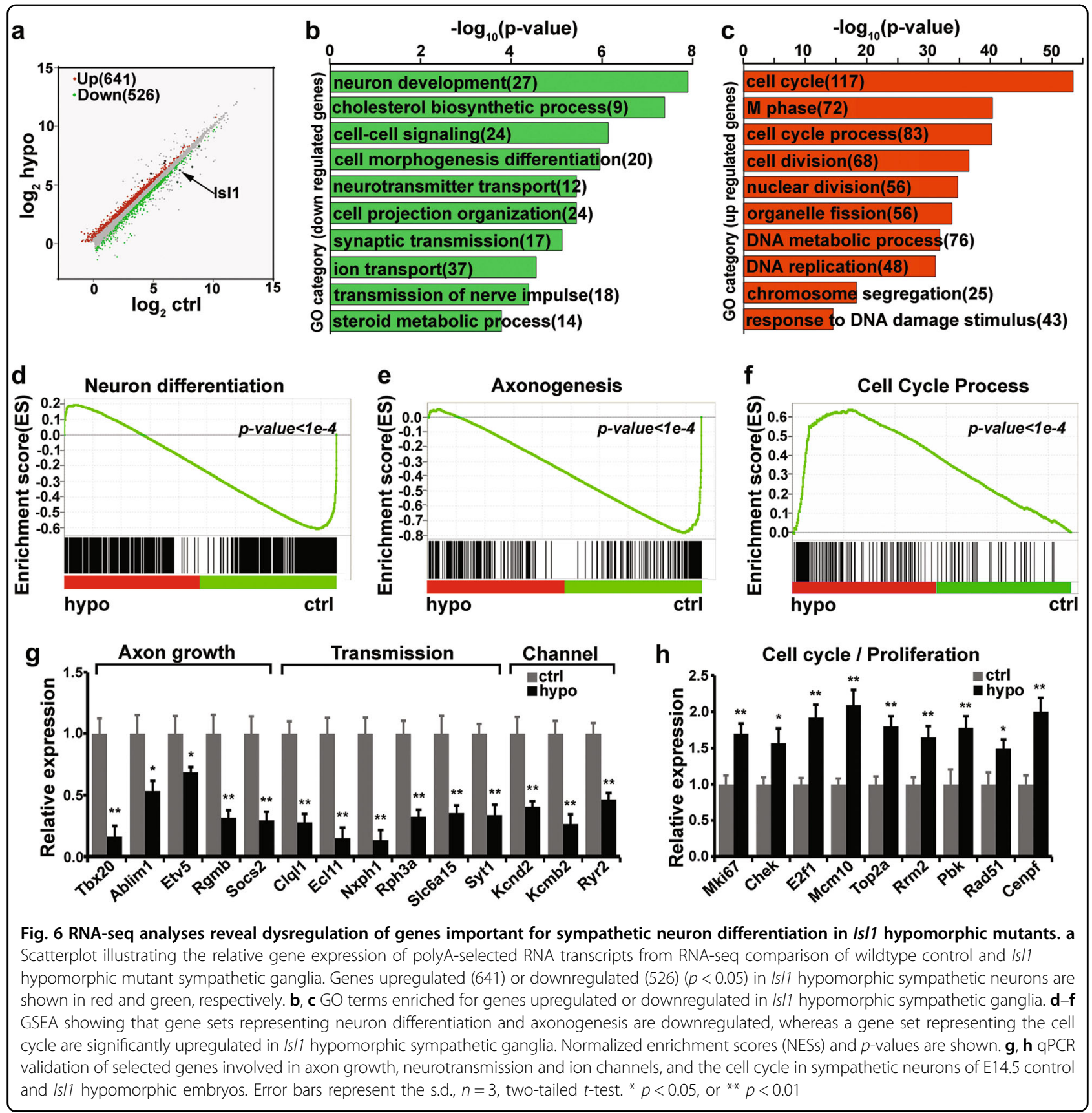

targets of ISL1 were involved in, e.g., cell adhesion and signal transduction (Fig. 8e, f; Supplementary Table 6). Similarly, for direct targets of ISL1 downregulated in E14.5 Isl1 hypomorphic mutants, over-represented categories included neuron differentiation and axonogenesis. For upregulated targets of ISL1, categories such as cell morphogenesis, neuron differentiation, and cell proliferation were prominent (Fig. 8h, i; Supplementary Table 7).

A close examination of direct targets of ISL1 downregulated in Isl1 CKO revealed a number of genes essential for sympathetic neuron development (e.g., Gata3, Insm1, Tlx3, Lin28b), axon growth, and neurotransmission (e.g., Bmpr1b, Plxna4, Snap25). Similarly, many direct targets of ISL1 downregulated in Isl1 hypomorphic sympathetic ganglia were involved in neuronal differentiation (e.g., Tbx20, Etv5, Phox2a) and neurotransmission (e.g., Ryr2, Kcnd2, Kcnmb2). Intersection of direct targets of ISL1 found in Isl1 CKO and hypomorphic mutants revealed 41 common targets of ISL1, many of which, e.g., $L m o 1, E g \ln 3, T b x 20$, were positively regulated by ISL1 at both early and late stages (Supplementary 

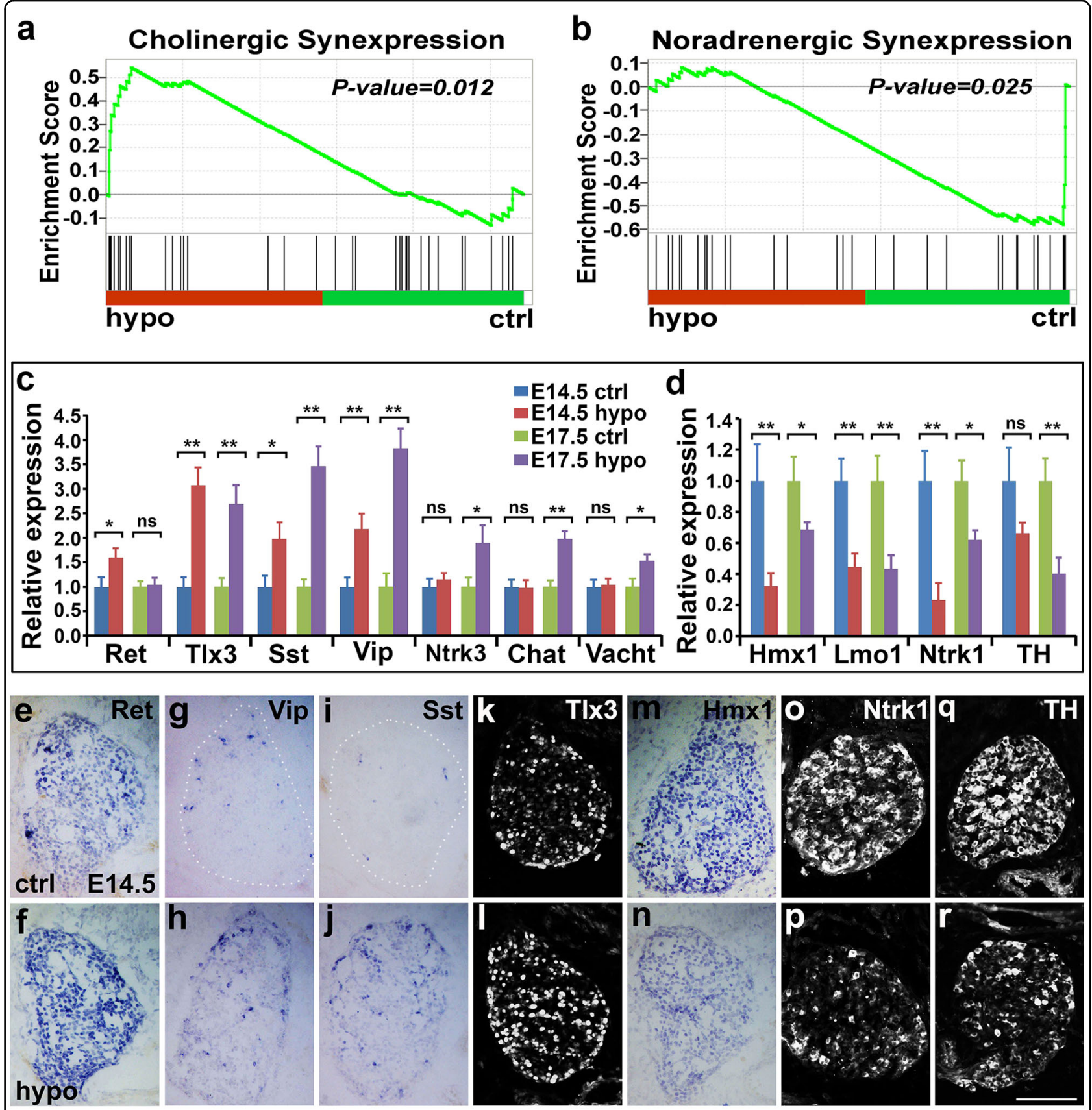

Fig. 7 Decreased noradrenergic and increased cholinergic traits in Is/1 hypomorphic mutant embryos. a, b GSEA showing downregulation of a gene set representing noradrenergic differentiation, but upregulation of a gene set representing cholinergic differentiation in $/ s / 1$ hypomorphic sympathetic ganglia. Normalized enrichment scores (NESs) and p-values are shown. c, d qPCR showing relative mRNA expression of noradrenergic and cholinergic neuron genes in Is/7 hypomorphic and control sympathetic ganglia at E14.5 and E17.5. Error bars represent the s.d., $n=3$, two-tailed t-test. e-r Verification of selected genes associated with cholinergic neurons (Ret, Vip, Sst, TIx3) and noradrenergic neurons (Hmx1, Ntrk1, TH) by in situ hybridization $(\mathbf{e}-\mathbf{j}, \mathbf{m}, \mathbf{n})$ and immunostaining $(\mathbf{k}, \mathbf{I}, \mathbf{o}-\mathbf{r})$ in E14.5 control and Is/1 hypomorphic sympathetic ganglia. Scale bar, $100 \mu \mathrm{m}$, ns $=$ not significant, ${ }^{*} p<0.05$, or ${ }^{* *} p<0.01$

Table 8). However, several direct targets of ISL1, such as Prox 1, Tlx3, and several cell cycle genes, were downregulated in Isl1 CKO during early development, but upregulated in Isl1 hypomorphic mutants during later development (Supplementary Table 8). Selected targets of ISL1 were verified by ChIP-qPCR, and genome browser views of representative common targets of ISL1 were shown (Fig. 8j and Supplementary Figure 5b). 


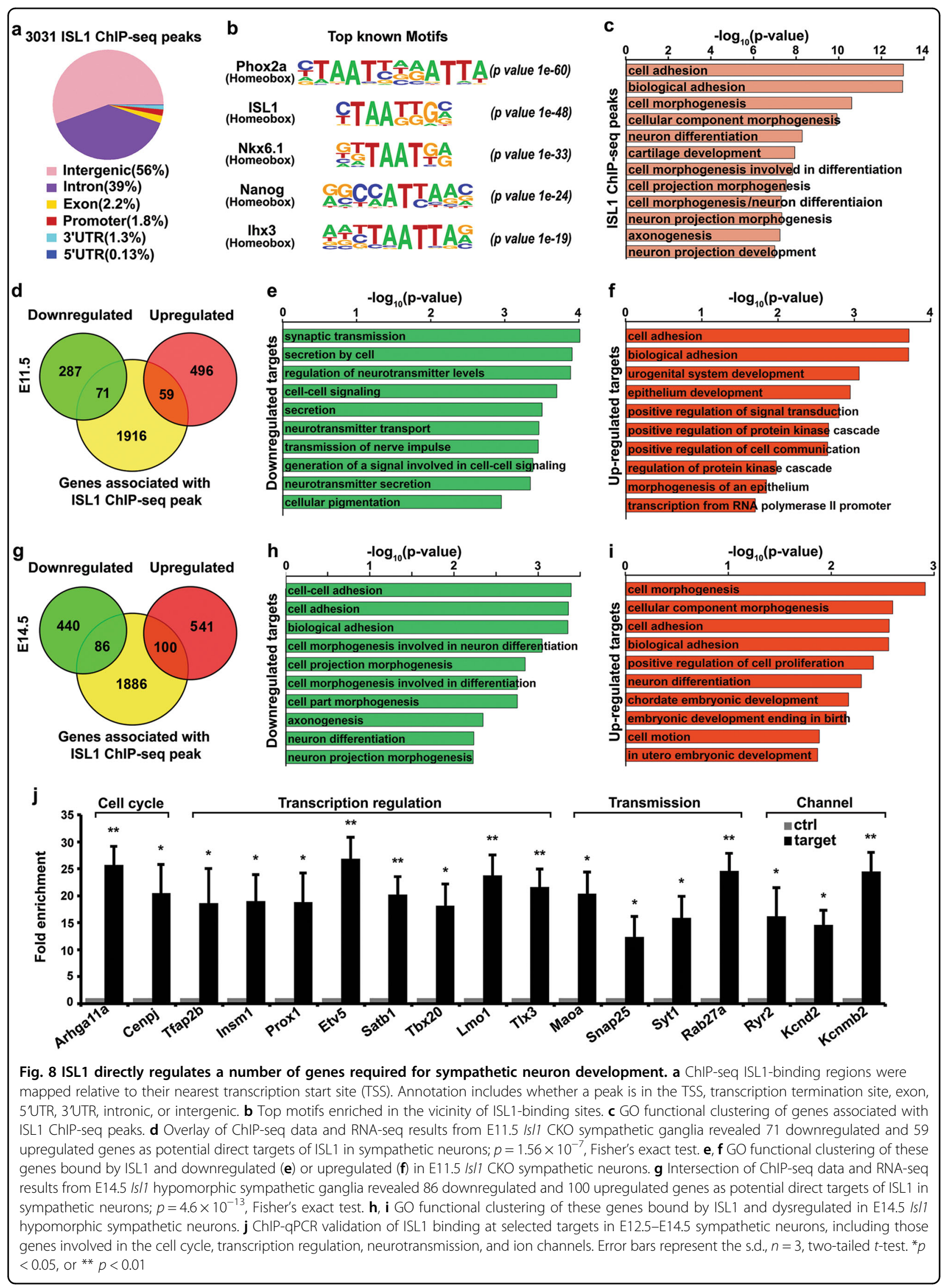


Notably, a number of targets of ISL1 downregulated in Isl1 CKO mutants were implicated in neuroblastoma pathogenesis $^{20-23}$, including Lmo1, Gata3, Prox1, Lin28b, and Alk. We found that ISL1 binds to an evolutionary conserved region within the first intron of Lmo1 (mm9: chr7:116289070-116289656) in mouse sympathetic neurons that, by whole-genome alignment, overlaps with the super-enhancer of LMO1 identified in human neuroblastoma ${ }^{20}$.

\section{Discussion}

\section{Distinct temporal requirements for ISL1 in sympathetic neuron proliferation and differentiation during sympathetic neuron development}

Sympathetic neurons continue to proliferate after the acquisition of pan-neuronal and noradrenergic properties $^{5}$. From E14.5 onward, a majority of sympathetic neurons undergo cell cycle withdrawal and terminal differentiation and maturation; however, the mechanisms underlying these temporal events remain largely unknown. At early developmental stages, ablation of Isl1 resulted in reduced sympathetic neuron proliferation and reduced expression of cell cycle genes, including Ccnd1 and Foxm1. Expression of a number of transcription factors critical for sympathetic neuron proliferation was also downregulated in Isl1 CKO mutants ${ }^{24-27}$, including Gata3, Insm1, and Prox1. Although mechanisms by which these transcription factors control sympathetic neuron proliferation are unclear, these data suggest that ISL1 may regulate sympathetic neuron proliferation by controlling cell cycle gene expression.

Differentiation of noradrenergic and pan-neuronal phenotypes is controlled by an early gene regulatory network ${ }^{3}$, including Mash1, Phox2b/2a, Hand2, Gata2/3, and Insm1. We found that ISL1 is downstream of Phox2b, but upstream of Gata2/3, Insm1/2, and Hand1. ISL1 acts parallel to Hand2, since Hand2 expression is not changed in Isl1 CKO mutants and vice versa ${ }^{28}$. ISL1 is dispensable for initial noradrenergic differentiation, since expression of TH and DBH is not altered in Isl1 CKO mutants. But instead, ISL1 directly or indirectly regulates sets of genes essential for sympathetic neuron development, axon growth, and neurotransmission, suggesting that ISL1 is essential for generic sympathetic neuronal differentiation during early sympathetic neuron development.

Reduced Isl1 expression in Isl1 hypomorphic mice caused impaired sympathetic innervations, delayed cell cycle withdrawal, and reduced expression of genes involved in axon growth and neurotransmission, demonstrating a continued requirement for ISL1 in sympathetic neuron differentiation and maturation at later development stages. Analyses of ChIP-seq and RNAseq data sets from both Isl1 CKO and hypomorphic mutants revealed ISL1 regulates common, yet distinct gene programs during early and later sympathetic neuronogenesis. Tlx3, Prox 1, and several cell cycle-related genes were among the direct targets of ISL1 that were downregulated in Isl1 CKO but upregulated in Isl1 hypomorphic mutants, suggesting that ISL1 may act both as an activator and a repressor in a context-dependent manner during sympathetic neuron development. Interestingly, a comparison of ISL1 targets in different cell types (sympathetic neurons, cardiac progenitors ${ }^{29}$, and sinus node pacemakers ${ }^{30}$ ) revealed few, if any, common targets that could account for the observed phenotypes in each of these mutants, suggesting that the action of ISL1 is cellular context-dependent.

Molecular mechanisms of ISL1 in sympathetic neurons remain unknown. Interestingly, ablation of Isl1 causes downregulation of a number of epigenetic regulators $^{29,31,32}$ (Dnmt1, Kdm6a, and Satb1) critical for neuronal development. In the heart, ISL1 physically interacts with Kdm6a to promote H3K27me3 demethylation at the enhancers of target genes encoding key cardiac transcription factors ${ }^{29}$. It is tempting to speculate that ISL1 might regulate the expression of sympathetic developmental genes via epigenetic mechanisms.

\section{Essential role of ISL1 in coordination of initial neurogenesis and gliogenesis by modulating BMP and} Notch signaling pathways

BMP and Notch pathways are essential for coordinating progenitor maintenance and neuronal differentiation during sympathetic nneurogenesis ${ }^{6}$. Ablation of Isl1 resulted in increased Notch signaling but decreased BMP signaling. Accordingly, genes associated with $\mathrm{NC}$ and glial progenitors were significantly upregulated, suggesting that during initial sympathetic neurogenesis, ISL1 is required for coordination of lineage differentiation of sympathetic neurons and glia by modulating BMP and Notch pathways. Interestingly, previous studies have revealed a role of ISL1 in cell fate specification and determination in sensory neurons of the dorsal root ganglion and spinal motor neurons. Ablation of $I s l 1$ in NC cells leads to reduced expression of genes essential for nociceptive neuron differentiation, but a failure in developmental downregulation of genes characteristic of sensory progenitors ${ }^{15}$. In spinal motor neurons, reduced Isl1 expression leads to conversion of motor neurons to V2 interneurons, although underlying mechanism remains unknown $^{18}$.

\section{ISL1 is required for diversification of noradrenergic and cholinergic sympathetic neurons}

Segregation of initially bimodal sympathetic neurons into noradrenergic and cholinergic neurons occurs later during development and involves antagonistic actions between pro-cholinergic (Ntrk3/Ret/Tlx3) and pro- 
noradrenergic (Hmx1/Ntrk1) factors ${ }^{13,14}$. In Isl1 hypomorphic sympathetic ganglia, we observed significantly reduced expression of genes essential for noradrenergic differentiation (e.g., Hmx1, Lmo1, Ntrk1, TH), but increased expression of genes essential for cholinergic differentiation (Ret, Tlx3, Sst, Vip, Ntrk3, Chat, Vacht), demonstrating that ISL1 is required for repression of cholinergic characteristics and maintenance of noradrenergic characteristics during later developmental stages. Previous studies have shown that, in forebrain and spinal cholinergic motor neurons, ISL1, complexed with LHX3 or LHX8, directly binds to and activates the expression of genes of cholinergic traits ${ }^{33}$. However, we did not find direct binding of ISL1 to the enhancers/ promoters of Chat, Vacht, or other cholinergic genes.

\section{ISL1 and neuroblastoma}

Neuroblastoma is an aggressive pediatric tumor arising from developing sympathetic neurons. ISL1 is overexpressed in most types of neuroblastoma, particularly in poorly differentiated neuroblastomas that indicates a poor prognosis $^{34,35}$. ISL1 is also a minimal residual disease marker of neuroblastoma ${ }^{34}$. However, the role of ISL1 in neuroblastoma tumorigenesis is unknown. We found that a number of genes implicated in neuroblastoma pathogenesis are downstream of ISL1 (e.g., Lmo1, Ccnd1, Tfap2b, Prox1, Casz1, Foxm1, Lin28b, Ccnd1, and Alk) ${ }^{20-23,34,36-38}$, many of which are direct targets of ISL1. Notably, LMO1 is a neuroblastoma oncogene associated with more proliferative and aggressive phenotype ${ }^{39}$. A polymorphism within a super-enhancer in the first intron of LMO1 that preserves an evolutionarily conserved GATA factor binding motif predisposes to neuroblastoma. Knockdown of GATA3 results in decreased LMO1 expression and suppression of neuroblastoma cell growth ${ }^{20}$. We found that ISL1 binds to an evolutionary conserved region within the first intron of Lmo1 (chr7:116289070-116289656) in mouse sympathetic neurons that overlaps with the super-enhancer of LMO1 identified in human neuroblastoma, suggesting coregulation of LMO1 expression by ISL1 and GATA3 and a potential role of ISL1 in controlling neuroblastoma cell proliferation.

Taken together, our study uncovered a temporal requirement for ISL1 during sympathetic neurogenesis (Supplementary Figure 6), and implicated Isl1 as a candidate gene for neuroblastoma.

\section{Materials and methods \\ Transgenic mice}

Generation of Wnt1-Cre;Isl1 ${ }^{\mathrm{f} / \mathrm{f}}$ and Isl1 hypomorphic mice $\left(I s l 1^{f-n e o / f-n e o}\right)$ have been reported previously ${ }^{18}$. To facilitate the visualization and lineage tracing of sympathetic neurons, $I s l 1^{\mathrm{f} / \mathrm{f}}$ mice were crossed onto a Rosa26LacZ or -Tomato reporter background ${ }^{40}$. All the experiments involving mice were carried out in accordance with protocols approved by the Animal Committee of Tongji University School of Medicine (TJmed-010-10).

In situ hybridization, immunostaining, and X-gal staining

In situ hybridization, immunostaining, and $\mathrm{X}$-gal staining were performed as described ${ }^{30}$. Fragments amplified from mouse cDNA were used to generate the RNA probes for Insm2, Tlx3, Tfap2b, Hand1, and Lmo1. Riboprobe for mouse Vip, Sst, and Ret were kindly provided by Dr. Leping Cheng (Chinese Academy of Sciences). For BrdU staining, pregnant mice were injected with $300 \mu \mathrm{l}$ of $\mathrm{BrdU}(100 \mu \mathrm{g} / \mathrm{g}$ body weight, Sigma). For cell counting, sections of sympathetic ganglia of the appropriate developmental stages were cut at $10 \mu \mathrm{m}$, every fourth section was stained, and positive cells were counted. For proliferation, the total number of BrdU+ sympathetic neurons (Phox $2 \mathrm{~b}+$ ) were counted and expressed as percentage of total Phox $2 \mathrm{~b}+$ neurons. For cell death assay, the total number of Casp- $3+$ neurons (Tomato + ) were counted and expressed as percentage of total Tomato + neurons. At least 5-8 matched sections were analyzed and three samples per genotype per time point were analyzed. Detailed information of antibodies is available in the Supplementary material.

\section{Sympathetic ganglion dissection and culture}

Sympathetic ganglia were dissected from E11.5-12 embryos of genotype Wnt1-Cre;Isl1 f/f (CKO) and Wnt1$\mathrm{Cre}_{\mathrm{Isl1}}{ }^{+/+}$(ctrl) with Rosa26-tdTomato reporter background. SCGs of E12 Isl1 CKO and control embryos on Rosa-tdTomato background were cultured in matrigel (BD Biosciences) in media containing NGF $(20 \mathrm{ng} / \mathrm{ml})$ and NT3 $(20 \mathrm{ng} / \mathrm{ml})$. Detailed protocol of sympathetic ganglion dissection and culture are available in the Supplementary material.

\section{RNA-seq and ChIP-seq data analysis}

RNA-seq analyses with two biological repeats were performed as described ${ }^{30}$. ChIP-seq was performed as previously described ${ }^{41}$. The complete RNA-seq and ChIPseq data sets are available from the Gene Expressing Omnibus database (http://www.ncbi.nlm.nih.gov/geo/) under the accession number GSE93308. For experimental and data analysis details, see the Online Data Supplement.

\section{Statistical analysis}

All experiments were performed for at least three independent times and respective data were used for statistical analyses. Data were presented as mean \pm s.d., and a two-tailed $t$-test was used for two-group comparisons. Differences were considered statistically significant at a value of $p \leq 0.05^{*}, p \leq 0.01^{* *}$. For experimental details, see the online Supplementary material. 


\section{Acknowledgements}

We thank Dr. Leping Cheng for sharing the antibody of Tlx3 and Riboprobe of Vip, Sst, Ret; Dr. Brunet J. F. for antibody of Phox2b. This work was supported by grants from the National Natural Science Foundation of China (grant numbers $81700285,81521061,81370196,81670448,81570285)$ and grants from the National Institutes of Health (HL123747, HL119967).

\section{Author details}

'Key Laboratory of Arrhythmia, Ministry of Education, East Hospital, Tongji University School of Medicine, Shanghai, China. ${ }^{2}$ School of Life Sciences and Technology, Tongji University, Shanghai, China. ${ }^{3}$ Key Laboratory of Systems Biomedicine, Ministry of Education, Shanghai Centre for Systems Biomedicine, Shanghai Jiao Tong University, Shanghai, China. ${ }^{4}$ Seventh People's Hospital of Shanghai University of TCM, Shanghai, China. ${ }^{5}$ Department of Medicine, Department of Pharmacology, Skaggs School of Pharmacy, University of California San Diego, California, USA

\section{Conflict of interest}

The authors declare that they have no conflict of interest.

\section{Publisher's note}

Springer Nature remains neutral with regard to jurisdictional claims in published maps and institutional affiliations.

Supplementary Information accompanies this paper at (https://doi.org/ 10.1038/s41419-018-0283-9).

Received: 6 November 2017 Revised: 14 December 2017 Accepted: 22 December 2017

Published online: 14 February 2018

\section{References}

1. Cheung, N. K. \& Dyer, M. A. Neuroblastoma: developmental biology, cancer genomics and immunotherapy. Nat. Rev. Cancer 13, 397-411 (2013).

2. Kameda, Y. Signaling molecules and transcription factors involved in the development of the sympathetic nervous system, with special emphasis on the superior cervical ganglion. Cell. Tissue Res. 357, 527-548 (2014).

3. Rohrer, $\mathrm{H}$. Transcriptional control of differentiation and neurogenesis in autonomic ganglia. Eur. J. Neurosci. 34, 1563-1573 (2011).

4. Huber, K. The sympathoadrenal cell lineage: specification, diversification, and new perspectives. Dev. Biol. 298, 335-343 (2006).

5. Gonsalvez, D. G. et al. Proliferation and cell cycle dynamics in the developing stellate ganglion. J. Neurosci. 33, 5969-5979 (2013).

6. Morrison, S. J. et al. Transient Notch activation initiates an irreversible switch from neurogenesis to gliogenesis by neural crest stem cells. Cell 101, 499-510 (2000).

7. Mukhopadhyay, A., Jarrett, J., Chlon, T. \& Kessler, J. A. HeyL regulates the number of TrkC neurons in dorsal root ganglia. Dev. Biol. 334, 142-151 (2009).

8. Tsarovina, K., Schellenberger, J., Schneider, C. \& Rohrer, H. Progenitor cell maintenance and neurogenesis in sympathetic ganglia involves Notch signaling. Mol. Cell. Neurosci. 37, 20-31 (2008).

9. Dunwoodie, S. L., Henrique, D., Harrison, S. M. \& Beddington, R. S. Mouse DII3: a novel divergent Delta gene which may complement the function of other Delta homologues during early pattern formation in the mouse embryo. Development 124, 3065-3076 (1997).

10. Pattyn, A., Morin, X., Cremer, H., Goridis, C. \& Brunet, J. F. The homeobox gene Phox $2 \mathrm{~b}$ is essential for the development of autonomic neural crest derivatives. Nature 399, 366-370 (1999).

11. Ernsberger, U. The development of postganglionic sympathetic neurons: coordinating neuronal differentiation and diversification. Auton. Neurosci. 94, 1-13 (2001).

12. Spitzer, N. C. Neurotransmitter switching? No surprise. Neuron 86, 1131-1144 (2015).

13. Furlan, A., Lubke, M., Adameyko, I., Lallemend, F. \& Ernfors, P. The transcription factor $\mathrm{Hm} \times 1$ and growth factor receptor activities control sympathetic neurons diversification. EMBO J. 32, 1613-1625 (2013).
14. Huang, T. et al. Tlx3 controls cholinergic transmitter and Peptide phenotypes in a subset of prenatal sympathetic neurons. J. Neurosci. 33, 10667-10675 (2013).

15. Sun, Y. et al. A central role for Islet1 in sensory neuron development linking sensory and spinal gene regulatory programs. Nat. Neurosci. 11, 1283-1293 (2008).

16. Huber, K. et al. The LIM-Homeodomain transcription factor Islet-1 is required for the development of sympathetic neurons and adrenal chromaffin cells. Dev. Biol. 380, 286-298 (2013).

17. Wakamatsu, Y., Endo, Y., Osumi, N. \& Weston, J. A. Multiple roles of Sox2, an HMG-box transcription factor in avian neural crest development. Dev. Dyn. 229, 74-86 (2004).

18. Liang, $\mathrm{X}$. et al. Isl1 is required for multiple aspects of motor neuron development. Mol. Cell. Neurosci. 47, 215-222 (2011).

19. Apostolova, G. et al. Neurotransmitter phenotype-specific expression changes in developing sympathetic neurons. Mol. Cell. Neurosci. 35, (397-408 (2007).

20. Oldridge, D. A. et al. Genetic predisposition to neuroblastoma mediated by a LMO1 super-enhancer polymorphism. Nature 528, 418-421 (2015).

21. George, R. E. et al. Activating mutations in ALK provide a therapeutic target in neuroblastoma. Nature 455, 975-978 (2008).

22. Becker, J., Wang, B., Pavlakovic, H., Buttler, K. \& Wilting, J. Homeobox transcription factor Prox 1 in sympathetic ganglia of vertebrate embryos: correlation with human stage 4s neuroblastoma. Pediatr. Res. 68, 112-117 (2010).

23. Hennchen, M. et al. Lin28B and Let-7 in the Control of Sympathetic Neurogenesis and Neuroblastoma Development. J. Neurosci. 35, 16531-16544 (2015).

24. Tsarovina, K. et al. The Gata3 transcription factor is required for the survival of embryonic and adult sympathetic neurons. J. Neurosci. 30, 10833-10843 (2010).

25. Wildner, H., Gierl, M. S., Strehle, M., Pla, P. \& Birchmeier, C. Insm1 (IA-1) is a crucial component of the transcriptional network that controls differentiation of the sympatho-adrenal lineage. Development 135, 473-481 (2008).

26. Holzmann, J., Hennchen, M. \& Rohrer, H. Prox1 identifies proliferating neuroblasts and nascent neurons during neurogenesis in sympathetic ganglia. Dev. Neurobiol. 75, 1352-1367 (2015).

27. Furlan, A. et al. Visceral motor neuron diversity delineates a cellular basis for nipple- and pilo-erection muscle control. Nat. Neurosci. 19, 1331-1340 (2016).

28. Stanzel, S. et al. Distinct roles of hand2 in developing and adult autonomic neurons. Dev. Neurobiol. 76, 1111-1124 (2016).

29. Wang, Y. et al. ISL1 and JMJD3 synergistically control cardiac differentiation of embryonic stem cells. Nucleic Acids Res. 44, 6741-6755 (2016).

30. Liang, X. et al. Transcription factor ISL1 is essential for pacemaker development and function. J. Clin. Invest. 125, 3256-3268 (2015).

31. Balamotis, M. A. et al. Satb1 ablation alters temporal expression of immediate early genes and reduces dendritic spine density during postnatal brain development. Mol. Cell. Biol. 32, 333-347 (2012).

32. Frauer, C. et al. Different binding properties and function of CXXC zinc finger domains in Dnmt1 and Tet1. PLOS ONE 6, e16627 (2011).

33. Cho, H. H. et al. Isl1 directly controls a cholinergic neuronal identity in the developing forebrain and spinal cord by forming cell type-specific complexes. PLoS Genet. 10, e1004280 (2014).

34. Cheung, I. Y., Feng, Y., Gerald, W. \& Cheung, N. K. Exploiting gene expression profiling to identify novel minimal residual disease markers of neuroblastoma. Clin. Cancer Res. 14, 7020-7027 (2008).

35. Bourdeaut, F. et al. Cholinergic switch associated with morphological differentiation in neuroblastoma. J. Pathol. 219, 463-472 (2009).

36. Ikram, F. et al Transcription factor activating protein 2beta (TFAP2B) mediates noradrenergic neuronal differentiation in neuroblastoma. Mol. Oncol. 10, 344-359 (2016).

37. Liu, Z. et al. CASZ1, a candidate tumor-suppressor gene, suppresses neuroblastoma tumor growth through reprogramming gene expression. Cell Death Differ. 18, 1174-1183 (2011).

38. Wang, Z. et al. FoxM1 in tumorigenicity of the neuroblastoma cells and renewal of the neural progenitors. Cancer Res. 71, 4292-4302 (2011).

39. Wang, K. et al. Integrative genomics identifies LMO1 as a neuroblastoma oncogene. Nature 469, 216-220 (2011).

40. Zhuang, S. et al. Expression of Is 11 during mouse development. Gene. Expr. Patterns 13, 407-412 (2013).

41. Lara-Astiaso, D. et al. Immunogenetics. Chromatin state dynamics during blood formation. Science 345, 943-949 (2014). 